ORIGINAL ARTICLE

\title{
Presentation of vitamin D deficiency
}

\author{
S Ladhani, L Srinivasan, C Buchanan, J Allgrove
}

Arch Dis Child 2004;89:781-784. doi: 10.1136/adc.2003.031385

See end of article for authors' affiliations ....................

Correspondence to: Dr S Ladhani, Department of Paediatrics, Newham General Hospital, Glen Road, Plaistow, London E13 8RU, UK: drshamez@aol.com

Accepted 16 May 2004

\begin{abstract}
Aims: To describe the various ways in which vitamin $D$ deficiency presents in children in selected districts of London and to identify which factors, if any, determine the mode of presentation.

Methods: Retrospective review of patients presenting to Newham General, Royal London, Great Ormond Street, and King's College Hospitals between 1996 and 2001 with either hypocalcaemia or rickets caused by vitamin $D$ deficiency. Children with plasma 25 -hydroxyvitamin $D$ levels $<25 \mathrm{nmol} / \mathrm{l}(10 \mathrm{ng} / \mathrm{ml})$ were considered to have vitamin D deficiency.

Results: Sixty five children, mostly from Black or Asian ethnic minority groups, were identified, 29 of whom had hypocalcaemic symptoms. Seventeen of these had no radiological evidence of rickets. The remainder (48 children) had radiological evidence of rickets with or without clinical signs. Symptoms and signs reverted to normal in all cases with vitamin $D$ supplementation. All children who presented with symptomatic hypocalcaemia were aged either $<3$ or $>10$ years. There was a strong correlation between age at presentation and population growth velocity reference data.

Conclusions: Rickets remains a problem in the UK especially in "at risk" ethnic minority groups. Symptomatic hypocalcaemia is an important, but under-recognised presenting feature. Growth rate is likely to be an important factor in determining the mode of presentation. Unexplained hypocalcaemia should be attributed to vitamin D deficiency in "at risk" ethnic minority groups until proved otherwise.
\end{abstract}

$\mathrm{T}$ he past few decades have seen a resurgence of vitamin D deficiency rickets in developed countries, particularly in dark skinned infants who are exclusively breast fed for prolonged periods without additional vitamin supplements. ${ }^{1-5}$ Vitamin D and its metabolites are required for calcium absorption from the gut and adequate delivery to the bone to promote normal bone mineralisation. ${ }^{4}$ Vitamin D deficiency results in reduced serum calcium, which triggers secretion of parathyroid hormone (PTH) to release calcium and phosphorus from bone in an attempt to maintain normal serum calcium levels. ${ }^{4}$ The consequence is usually rickets in children, which is defined as failure of mineralisation (osteomalacia) of growing bone or osteoid tissue. Children with vitamin D deficiency classically present soon after weight bearing age with bony abnormalities such as bowing of the legs, knock-knees, rickety rosary of the costo-chondral junctions, swelling of the ends of long bones, frontal bossing of the skull, and pathological fractures in severe cases, as well as poor growth, delayed dentition, and slow motor development. ${ }^{4}{ }^{6}$ However, another group of children with vitamin D deficiency has been described, who present with symptoms of hypocalcaemia (neuromuscular irritability, including convulsions, tetany, and paraesthesia) with or without radiological evidence of rickets; ${ }^{5-11}$ it has been suggested that this is an early phase (stage 1 ) of vitamin D deficiency which usually occurs in young infants below 6 months of age. ${ }^{12}$ To date, there have been no studies to identify any differences between these two groups that might explain such contrasting presenting features.

\section{METHODS}

The case records of children aged 0-16 years at the time of diagnosis with vitamin D deficiency treated at three northeast London hospitals-Newham General Hospital, Royal London, and Great Ormond Street-or King's College Hospital, in southeast London, between 1996 and 2001 were studied retrospectively. A predefined questionnaire was used to extract relevant information from the case notes. Calcium, phosphate, and alkaline phosphatase (ALP) levels were measured by standard methods (Olympus AU600, Hamburg) in all laboratories. Vitamin D status, as determined by measurement of 25-hydroxyvitamin D (25-OHD), and intact parathyroid hormone (PTH) levels were determined by radioimmunoassay (ImmunoDiagnostic Systems, UK) and two site radioimmunometric assay (Nicholl Institute Diagnostic Kit, USA), respectively, using commercially available diagnostic kits. Children with plasma 25-OHD levels less than $25 \mathrm{nmol} / \mathrm{l}(10 \mathrm{ng} / \mathrm{ml})$ were considered to have vitamin D deficiency. The normal range for PTH is 1.1-6.8 pmol/l. The presence or absence of radiological evidence of rickets was determined from routine radiological reports.

The diagnosis of rickets was based on a combination of clinical findings (bow legs, rickety rosary, bone pain or deformity, tetany, convulsions due to hypocalcaemia), radiological evidence, biochemistry results (raised alkaline phosphatase with or without raised PTH) or low serum 25OHD level, with a positive response to treatment with oral vitamin D and calcium supplementation. Children with hepatic or renal abnormality or on anticonvulsant therapy (including one who had concomitant vitamin D deficiency) were excluded from the study. Data were entered into Microsoft Excel and statistical analysis was performed using Stata, version 7.0.

Since this was a retrospective study using only data that had been collected for clinical purposes, ethical committee approval was not obtained.

\section{RESULTS}

A total of 65 children were identified with vitamin D deficiency rickets during the study period. Two other children with renal tubular rickets were excluded. Thirty nine children were of Asian origin, 24 Afro-Caribbean, and two Eastern European. There was little difference in the characteristics of children diagnosed in the two London regions, apart from the observation that 13/19 of children at King's College Hospital were Afro-Caribbean compared to $10 / 46$ presenting to the north London hospitals, while $36 / 46$ of children presenting to 
Table 1 Main presenting complaints among the 65 children with vitamin $D$ deficiency

\begin{tabular}{lrlr}
\hline $\begin{array}{l}\text { Hypocalcaemic } \\
\text { symptoms }(\mathbf{n = 2 9 )}\end{array}$ & $\mathbf{n}$ & $\begin{array}{l}\text { No hypocalcaemic } \\
\text { symptoms }(\mathbf{n = 3 6})\end{array}$ & $\mathbf{n}$ \\
\hline Convulsions & 16 & Incidental & 11 \\
Neuromuscular irritability & 9 & Bow legs & 10 \\
Apnoea & 3 & Bone pain & 4 \\
Stridor & 1 & Swollen joints & 3 \\
& & Bone abnormality & 2 \\
& & Motor delay & 2 \\
& & Short stature & 2 \\
& & Screening family member & 2 \\
\hline & & \\
& & & \\
& &
\end{tabular}

the latter hospitals were Asian compared to 3/19 at King's College Hospital. There were 37 (45\%) males.

Almost half the children $(29 / 65 ; 45 \%)$ presented with symptoms of hypocalcaemia (table 1). The remainder presented with symptoms and signs of bony rickets (fig 1). There was no difference in sex for the two modes of presentation. The 25-OHD concentrations of the patients are shown in table 2 . All had concentrations below $15 \mathrm{nmol} / \mathrm{l}$.

Children presenting with hypocalcaemic symptoms had significantly lower median calcium and alkaline phosphatase levels, and higher median phosphate levels, but there was no significant difference in PTH levels (table 2). Only 12/29 $(41 \%)$ children presenting with hypocalcaemic symptoms had radiological evidence of rickets compared to 35/36 (97\%) who did not (one girl with Neiman-Pick type C disorder was incidentally discovered to have hypocalcaemia secondary to nutritional vitamin D deficiency on routine blood tests with no radiological evidence of rickets; she successfully responded to treatment with oral vitamin $\mathrm{D}$ ).

The most striking difference between the two modes of presentation was the age distribution (fig 2). ${ }^{13}$ Children presenting with hypocalcaemic symptoms were exclusively either under 3 or over 10 years of age. In particular, 9 of 11 $(82 \%)$ infants under the age of 6 months, 16 of $23(70 \%)$ under 1 year, and all seven children over the age of 10 years presented with hypocalcaemic symptoms. In contrast, vitamin D deficiency manifest as rickets without symptomatic
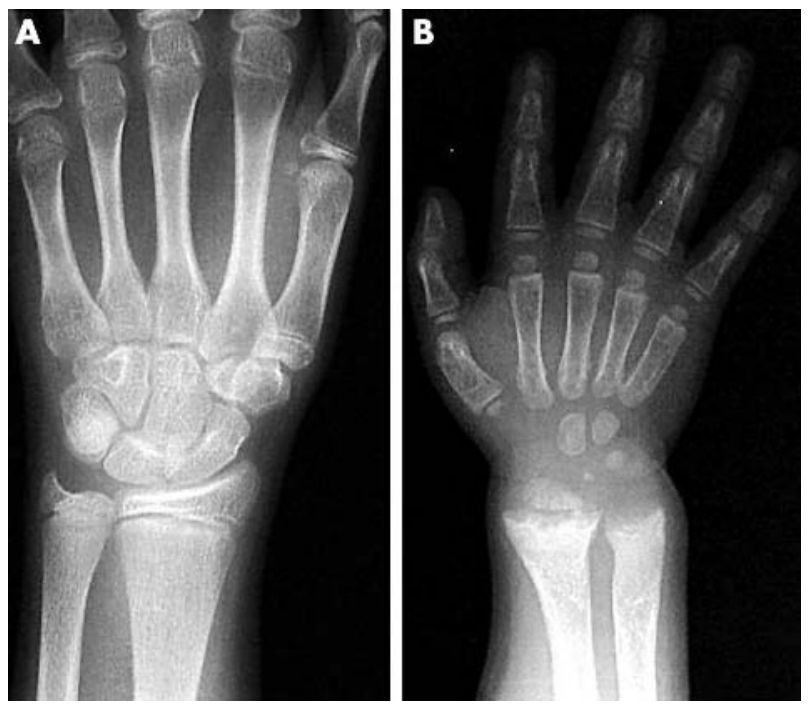

Figure 1 (A) Radiograph of patient aged 1 year with severe rickets. (B) Radiograph of patient aged 11 years presenting with hypocalcaemic symptoms and no radiological evidence of rickets.
Table 2 A comparison of children presenting with and without hypocalcaemic symptoms

\begin{tabular}{llll}
\hline & $\begin{array}{l}\text { Hypocalcaemic } \\
\text { symptoms }(\mathbf{n}=29)\end{array}$ & $\begin{array}{l}\text { No hypocalcaemic } \\
\text { symptoms }(\mathbf{n}=36)\end{array}$ & p value \\
\hline $\mathrm{Ca}^{2+}(\mathrm{mmol} / \mathrm{l})$ & $1.36(0.84-2.08)$ & $2.11(1.32-2.49)$ & $<0.0001$ \\
$\mathrm{PO}_{4}(\mathrm{mmol} / \mathrm{l})$ & $1.57(0.85-3.16)$ & $0.87(0.38-4.21)$ & $<0.0001$ \\
$\mathrm{ALP}(\mathrm{IU} / \mathrm{l})$ & $989(266-4393)$ & $1723(358-8988)$ & $<0.01$ \\
$25-\mathrm{OHD}$ & $5.0(2.1-14)$ & $6.7(2.7-14)$ & 0.48 \\
$(\mathrm{nmol} / \mathrm{l})$ & & & \\
$\mathrm{PTH}(\mathrm{pmol} / \mathrm{l})^{*}$ & $177(22-760)$ & $258(21-640)$ & 0.59 \\
\hline
\end{tabular}

Results presented as median (range).

*Parathyroid hormone levels were only available for 18 and 14 children with and without hypocalcaemic symptoms, respectively (normal range: $1.1-6.8 \mathrm{pmol} / \mathrm{l})$.

hypocalcaemia, presented across the range of prepubertal ages, with the majority under 3 years of age.

Both modes of presentation occurred at all months of the year, but children presenting with hypocalcaemia showed a peak in the months following winter (March to July), while children with rickets presented almost equally throughout the year (fig 3). All children responded to treatment with vitamin $\mathrm{D}$ and their biochemical abnormalities returned to normal. One child with coeliac disease responded to a glutenfree diet with vitamin D supplementation.

\section{DISCUSSION}

In the absence of any renal tubular disorder or defect in vitamin D metabolism, vitamin D deficiency usually results from what is usually referred to as nutritional deficiency. Rickets may also occur as a result of calcium deficiency ${ }^{14}$ and this could possibly have been an additional factor in our patients. However, since vitamin D is principally derived from the action of sunlight on exposed skin, and darker skinned individuals require more exposure to sunlight than lighter skinned individuals to manufacture adequate vitamin $\mathrm{D},{ }^{15}$ any deficiency must be made up from dietary sources. Consequently, although vitamin D deficiency should strictly be regarded as a failure of adequate sunlight exposure, ${ }^{16}$ it is usually referred to as being "nutritional".

In young infants, maternal vitamin $\mathrm{D}$ deficiency resulting in poor materno-fetal transfer of vitamin D during pregnancy and prolonged breast feeding are added risk factors. High phytic acid dietary content among some Asian children, particularly those whose diet contains a high proportion of chapattis, may also be an important aetiological factor, ${ }^{4-6}$ contributing to both vitamin $\mathrm{D}$ and calcium deficiency.

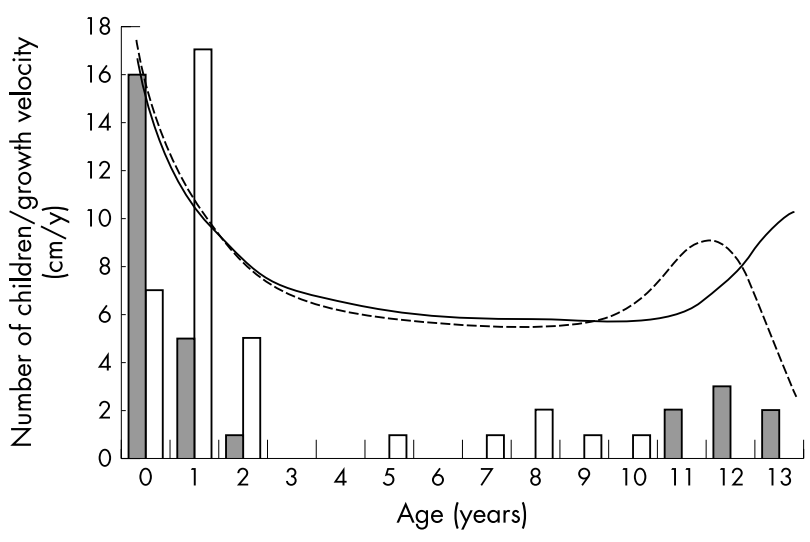

Figure 2 Age of children with vitamin D deficiency presenting with (dark bars) and without (light bars) hypocalcaemic symptoms. Growth velocity lines ${ }^{12}$ for boys (solid line) and girls (dotted line) have been superimposed onto the graph. 


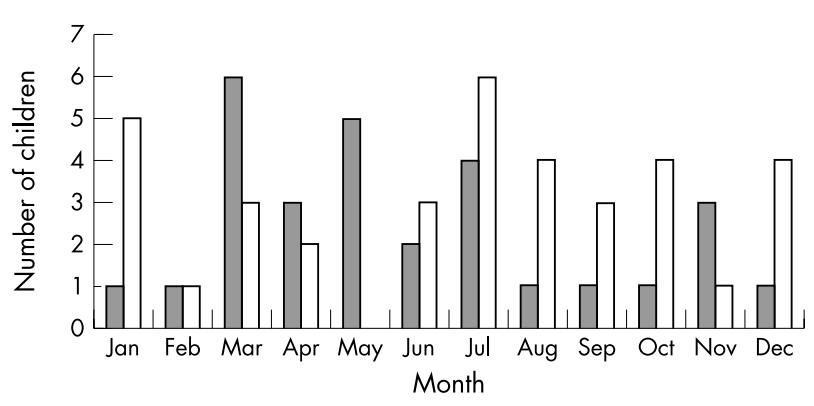

Figure 3 Month of presentation for children with (dark bars) and without (light bars) hypocalcaemia.

We have presented 65 cases of vitamin D deficiency in children managed by hospital paediatric departments over a five year period. Vitamin D deficiency remains the commonest cause of newly presenting hypocalcaemia in our units outside the neonatal period and in all but one case, no underlying pathological cause of the vitamin D deficiency was identified. In this case, a 12 year old Asian girl who did not initially respond to vitamin D supplementation, coeliac disease was confirmed by jejunal biopsy. All of the patients had low levels of vitamin $\mathrm{D}$ at diagnosis and all responded to treatment with vitamin D and calcium supplementation.

The children presented during all months of the year and not mainly during the post-winter months as has been described previously. ${ }^{3}$ Indeed, the month in which most children were diagnosed with vitamin D deficiency was July, which is the peak of summer in the UK. Almost half the children presented with symptoms of hypocalcaemia and, of these, fewer than half had radiological evidence of rickets.

Presentation with hypocalcaemic symptoms was restricted to two age groups-those under 3 or over 10 years of age. Vitamin D deficiency has been described as occurring in three stages, ${ }^{12}$ with hypocalcaemia occurring mainly in stage 1. Symptoms of hypocalcaemia in the age groups reported here have previously been described. Felner and colleagues 9 reported three children, aged 6 weeks, 9 weeks, and 3 years, with nutritional vitamin D deficiency, who presented with acyanotic generalised tonic-clonic seizures (two cases) or carpopedal spasm (one case) and were found to have hypocalcaemia, hyperphosphataemia, raised PTH levels, and normal radiographs - all responded to treatment with vitamin D. Pal ${ }^{5}$ reported nine cases of symptomatic hypocalcaemia in young infants, more than half of whom were under 5 months of age, while Binet and Kooh ${ }^{15}$ reported that two of their 17 patients with vitamin D deficiency aged 7-33 months presented with hypocalcaemic fits.

There are several reports of adolescents with rickets who presented with hypocalcaemia. Narchi and colleagues ${ }^{10}$ reported 21 teenagers aged $10-15$ years with rickets presenting to their local health centre in Saudi Arabia over a two year period; 12 (57\%) had carpopedal spasm and only eight (38\%) had radiological evidence of rickets. Nutritional rickets in adolescents has also been reported in Asians and North Africans living in developed countries with a colder climate. ${ }^{11}$

The age of children presenting with symptomatic hypocalcaemia correlates well with the periods of rapid growth (fig 2). Although we do not have individual growth velocity data on our patients, the strong correlation with normal population growth rates suggests that growth rate is an important factor in determining the mode of presentation and we propose that the hypocalcaemia is attributable to the increased metabolic demands of rapid growth during these periods, ${ }^{11}{ }^{13}$ resulting in development of symptomatic hypocalcaemia before any radiological changes occur. If left untreated, these patients may later present with rickets.
This theory is consistent with the significantly higher ALP levels and asymptomatic hypocalcaemia or normocalcaemia in children who presented with rickets. In these children, a lower metabolic demand for calcium would result in a more chronic course of disease, poor mineralisation of new bone, and increasing osteomalacia. On the other hand, the high metabolic demand for calcium in children presenting with hypocalcaemic symptoms results in a more acute presentation, before radiological evidence of rickets can occur.

Another explanation for the hypocalcaemia observed is that children with vitamin D deficiency and high levels of PTH become PTH resistant and, therefore, remain hypocalcaemic. ${ }^{17}$ However, although PTH resistance is a feature of vitamin D deficiency, this is unlikely to be the explanation for the differences seen because children presenting with hypocalcaemic symptoms had lower median PTH levels, although this was not statistically significant (table 2). However, it would help to explain the hyperphosphataemia seen in these patients.

The proportion of children with vitamin D deficiency presenting with hypocalcaemia in our study $(45 \%)$ is higher than other reports. Narchi's group, ${ }^{10}$ for example, reported that only $12 / 21(57 \%)$ adolescents aged $10-15$ years presented with hypocalcaemic symptoms in contrast to $7 / 7$ (100\%) in our study; and 2/18 (11\%) children 5-25 months of age reported by Kreiter's group ${ }^{18}$ presented with hypocalcaemia compared to $13 / 39$ (33\%) children in the same age group in our study. This is probably because many high risk children (dark skinned, prolonged breast feeding, maternal vitamin D deficiency) and mild cases of rickets are usually treated with vitamin D supplements by the general practitioner in the community or paediatric outpatient clinics. In contrast, children with hypocalcaemic symptoms are more likely to present to the hospital and be admitted for investigation and treatment.

\section{Conclusion}

Vitamin D deficiency has clearly re-emerged as a problem in the UK, especially in "at risk" ethnic minority groups. A significant number of children with vitamin D deficiency present with symptoms of hypocalcaemia with no radiological evidence of rickets. This is an under-recognised feature of vitamin D deficiency. Infants, toddlers, and adolescents are at particularly high risk, probably through the increased metabolic demand of rapid growth during these periods. Vitamin D deficiency should always be considered the likeliest cause of hypocalcaemia in these groups. This study emphasises the importance of vitamin D supplementation, not only in at risk infants but also in adolescents.

\section{Authors' affiliations}

S Ladhani, L Srinivasan, J Allgrove, Department of Paediatrics, Newham General Hospital, London, UK

C Buchanan, Department of Paediatrics, King's College Hospital, London, UK

\section{REFERENCES}

1 Shaw NJ, Pal BR. Vitamin D deficiency in UK Asian families: activating a new concern. Arch Dis Child 2002;86:147-9.

2 Welch TR, Bergstrom WH, Tsang RC. Vitamin D-deficient rickets: the reemergence of a once-conquered disease. J Pediatr 2000;137:143-5.

3 Lawson M, Thomas M. Vitamin D concentrations in Asian children aged 2 years living in England: population survey. BMJ 1999;318:28.

4 Thomas MK, Demay MB. Vitamin D deficiency and disorders of vitamin D metabolism. Endocrinol Metab Clin N Am 2000;29:611-27.

5 Pal BR. Rickets Resurgence in the United Kingdom: improving antenatal management in Asians. J Pediatr 2001;139:337-8.

6 Mughal MZ, Salama H, Laing I, et al. Florid rickets associated with prolonged breast feeding without vitamin D supplementation. BMJ 1999;318:39-40.

7 Duplechin RY, Nadkarni M, Schwartz RP. Hypocalcaemic tetany in a toddler with undiagnosed rickets. Ann Emerg Med 1999;34:399-402. 
8 Halterman JS, Smith SA. Hypocalcaemia and stridor: an unusual presentation of vitamin D-deficient rickets. J Emerg Med 1998;16:41-3.

Felner El, Marks JF, Germak JA. A variation of vitamin D deficiency in children. J Pediatr Endocrinol Metab 2001;14:203-6.

10 Narchi H, El Jamil M, Kulaylat N. Symptomatic rickets in adolescence. Arch Dis Child 2001;84:501-3.

11 Moncrief MW, Lunt HRW, Arthur LH. Nutritional rickets at puberty. Arch Dis Child 1973;48:221-4

12 Fraser D, Kooh SW, Scriver CR. Hyperparathyroidism as the cause of hyperaminoaciduria and phosphaturia in human vitamin D deficiency. Pediatr Res 1967; 1:425-35.

13 Tanner JM, Whitehouse RH. Clinical longitudinal standards for height, weight, height velocity, weight velocity and stages of puberty. Arch Dis Child 1976;51:170-9.
14 Delucia MC, Minick ME, Carpenter TO. Nutritional rickets with normal circulating 25-hydroxyvitamin D: a call for reexamining the role of dietary calcium intake in North American infants. J Clin Endocrinol Metab 2003;88:3539-45.

15 Binet A, Kooh SW. Persistence of vitamin D-deficiency in Toronto in the 1990s. Can J Public Health 1996;87:227-30.

16 Lo CW, Paris PW, Holick MF. Indian and Pakistani immigrants have the same capacity as Caucasian to produce vitamin D in response to ultraviolet irradiation. Am J Clin Nutr 1986;44:683-5.

17 Allgrove J, Chayen J, Jayaweera P, et al. An investigation of the biological activity of parathyroid hormone in pseudohypoparathyroidism: comparison with vitamin D deficiency. Clin Endocrinol (Oxf) 1984;20:503-14.

18 Kreiter SR, Schwartz RP, Kirkman HN Jr, et al. Nutritional rickets in African American breast-fed infants. J Pediatr 2000;137:153-7.

\section{IMAGES IN PAEDIATRICS}

\section{Angioinvasive aspergillosis}

Collowing chemotherapy for leukaemia, a - 6 year old girl developed multiple necrotic mucosal and skin lesions from which

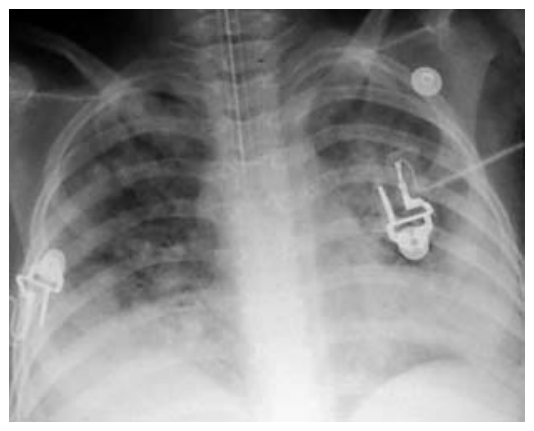

Figure 1
Aspergillus flavus was cultured. A chest radiograph (fig 1) revealed bilateral consolidation attributed to either alveolar haemorrhage or

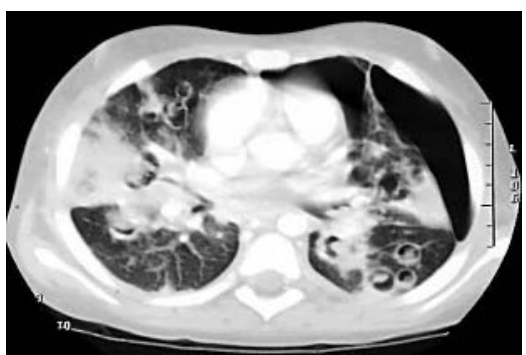

Figure 2 aspergillosis. A subsequent computed tomography (CT) scan (fig 2) revealed central cavitation and a unilateral pneumothorax. In invasive aspergillosis initial cavitation, thin ground glass haloes and small pneumothoraces are better detected by CT scan. ${ }^{1}$

V K T Nootigattu, J Sundaram, A Shabbani, S D Playfor

Royal Manchester Children's Hospital, Manchester, UK; ntirupatayya@yahoo.com

\section{Reference}

1 Sergiacomi G, Tomiselli A, Grimaldi P, et al. Invasive aspergillosis in the haematologic patient: the usefulness of computed tomographic and high-resolution computed tomographic studies. Radiologia Medica 1996;91:377-84. 